Comunicações Breves

Brief Communication

\author{
Anna Beatriz de Paiva Gomes ${ }^{1}$ (C) \\ Marcia Simões-Zenari ${ }^{12}$ \\ Kátia Nemr ${ }^{1,2}$
}

Descritores

Envelhecimento Voz Laringe Disfonia Distúrbios da Voz Qualidade da Voz

Keywords Aging Voice Larynx Dysphonia Voice Disorders Voice Quality

Endereço para correspondência:

Marcia Simões-Zenari

Curso de Fonoaudiologia,

Departamento de Fisioterapia,

Fonoaudiologia e Terapia Ocupacional, Faculdade de Medicina, Universidade de São Paulo - USP

Rua Cipotânea, 51, Cidade

Universitária, São Paulo (SP), Brasil, CEP: 05360-000.

E-mail: marciasz@usp.br

Recebido em: Abril 24, 2020.

\section{Voz do idoso: o avanço da idade gera diferentes impactos?}

\section{Aged voice: does advancing age generate different impacts?}

\section{RESUMO}

Objetivo: Verificar se existem diferenças em aspectos vocais entre idosos com presbifonia, divididos em três faixas etárias. Método: Participaram 60 idosos de ambos os sexos com idades entre 60 e 90 anos (média=72,3), com diagnóstico de presbifonia estabelecido em avaliação otorrinolaringológica. A partir da gravação das vozes foi realizada análise perceptivo-auditiva e acústica e os dados foram comparados por meio dos testes estatísticos com os participantes divididos nos grupos etários 60-70 anos, 71-80 anos e 81-90 anos. Resultados: Ainda que os idosos dos três grupos tenham apresentado alterações em aspectos vocais variados, como instabilidade e ruído em frequências graves, aqueles com mais de 80 anos apresentaram maior grau de disfonia, rugosidade, soprosidade e pitch. Também foram observados neste grupo maiores desvios em jitter, shimmer e irregularidade e a medida GNE no limite da normalidade. Todas as diferenças foram estatisticamente significativas. A maioria dos idosos dessa faixa etária apresentou ainda alteração no diagrama de desvio fonatório e quebra de frequência. Conclusão: Diversos aspectos perceptivo-auditivos e acústicos se apresentaram mais desviados nos idosos mais velhos, o que reforça a necessidade de serem consideradas essas especificidades tanto na avaliação dos efeitos do envelhecimento na voz quanto no desenvolvimento de ações para minimizar o declínio vocal.

\begin{abstract}
Purpose: To verify if there are differences in the vocal aspects of older people from three different age groups with presbyphonia diagnosis. Methods: Sixty older adults joined this study. They were both female and male, with an age range from 60 to 90 years old (average: 72.3) and with presbyphonia diagnosis established after otolaryngology evaluation. From their voice recordings, it was possible to make the acoustic and auditory-perceptual analysis. The data collected was compared through statistical tests considering the division of the participants into the following groups: 60-70 years old, 71-80 years old, and 81-90 years old. Results: Even though the older people from all of the three groups have presented deviation in multiple vocal aspects such as instability and vocal noise in low frequencies, those with more than 80 years old have presented a higher deviation of the general grade of dysphonia, roughness, breathiness, and pitch. In this group, it was also observed higher deviations in jitter, shimmer, vocal breaks, and the GNE measure on the edge of normality. All the differences were statistically significant. The majority of the older participants from that group presented even a deviation in the phonatory deviation diagram and frequency break. Conclusion: Various acoustic and auditory-perceptual aspects had a higher deviation in the older adults over 80 years old, which reinforces the need to consider those specificities in the evaluation of the vocal aging impacts and also in the development of actions to minimize vocal declination.
\end{abstract}

Trabalho realizado na Universidade de São Paulo - USP - São Paulo (SP), Brasil.
${ }^{1}$ Curso de Especialização em Voz, Escola de Educação Permanente, Hospital das Clínicas, Faculdade de
Medicina, Universidade de São Paulo - USP - São Paulo (SP), Brasil.
${ }^{2}$ Curso de Fonoaudiologia, Departamento de Fisioterapia, Fonoaudiologia e Terapia Ocupacional, Faculdade
de Medicina, Universidade de São Paulo - USP - São Paulo (SP), Brasil. Fonte de financiamento: nada a declarar.

Conflito de interesses: nada a declarar. 


\section{INTRODUÇÃO}

Com o avanço da idade ocorre gradativa deterioração das estruturas laríngeas e de toda fisiologia responsável pela fonação ${ }^{(1,2)}$, com impacto em aspectos vocais perceptivo-auditivos e acústicos ${ }^{(3)}$.

O declíno muscular do processo de envelhecimento faz com que pacientes com presbifonia apresentem arqueamento das pregas vocais, com formação de fenda glótica durante a fonação ${ }^{(1,4)}$. As cartilagens aritenoides são mais proeminentes do que em adultos e jovens e observa-se assimetria ou redução da amplitude da onda mucosa, além de predominância da fase aberta no ciclo vibratório das pregas vocais ${ }^{(4,5)}$. Somam-se a isso mudanças microscópicas na camada superficial da lâmina própria que podem incluir espessamento ou edema, atrofia de fibras elásticas e diminuição do número de fibroblastos. Histologicamente há queda do número total de células, mudanças na síntese proteica e redução da produção de matriz extracelular ${ }^{(5)}$.

O envelhecimento impacta também nos mecanismos de respiração e de ressonância, e as comorbidades e uso de medicamentos observados com frequência nos idosos interferem na produção vocal como um todo ${ }^{(6)}$. A voz envelhecida pode apresentar harmônicos reduzidos, alteração da frequência fundamental, aumento de jitter e shimmer, extensão vocal restrita, incoordenação pneumofonoarticulatória, aumento da soprosidade, rugosidade e tremor, dificuldades de modulação da intensidade, além da diminuição da resistência vocal e dos tempos máximos fonatórios ${ }^{(7)}$.

Com o avanço da idade, o metabolismo se torna mais lento e o funcionamento endócrino é reduzido, principalmente após a menopausa, no caso das mulheres. Os tecidos glandulares são progressivamente atrofiados e isso pode gerar redução da extensão vocal e alterações na frequência fundamental - diminuição nas mulheres e elevação nos homens. Isso faz com que as vozes de ambos os sexos possam ser confundidas com mais facilidade na velhice do que na juventude ou na vida adulta ${ }^{(8)}$. As queixas relatadas pelos idosos podem ser voz fraca e soprosa ${ }^{(9)}$, resistência vocal diminuída e rouquidão ${ }^{(1)}$, além de dificuldade para projetar a $\mathrm{Voz}^{(9)}$, ser ouvido na presença de ruído ${ }^{(1)} \mathrm{e}$ ter que repetir muitas vezes o que falam ${ }^{(2)}$. Fatores estes que impactam diretamente em sua comunicação e sociabilidade, ainda que em graus variados ${ }^{(5)}$. Prejuízos na comunicação abalam o bem-estar psicológico e a autonomia dos idosos ${ }^{(2,6)}$.

Estima-se que a prevalência de distúrbios de voz em idosos varie entre 4,8\% e 39,3\% $\%^{(1,2,9)}$. Kost e Sataloff ${ }^{(5)}$ descrevem mudanças decorrentes do envelhecimento mais significativas na laringe dos homens ${ }^{(5)}$. Ressalte-se que o processo de envelhecimento tem mudado com o avanço da ciência, da tecnologia e dos hábitos de vida, o que impacta no aumento da longevidade. Em apenas dez anos o número de idosos cresceu em 40,3\% no Brasil e observou-se diminuição de internações hospitalares deste grupo, o que pode ser resultado da expansão dos serviços de atenção básica à saúde e melhora da sua qualidade ${ }^{(10)}$. Neste cenário, é fundamental considerar que os idosos estão cada vez mais heterogêneos, e não é suficiente elaborar estratégias em saúde considerando-os apenas como um único grupo de indivíduos acima de 60 anos. Inclusive as condições de saúde podem ser muito diversas entre idosos de diferentes idades ${ }^{(2)}$. Afora isso, o uso profissional da voz é algo que perdura para vários deles; nesses casos, além de sinais de presbilaringe e de quadros em graus variados de presbifonia, pode-se ter em associação outros comprometimentos vocais como sinais e sintomas relacionados ao fonotrauma, por exemplo.

O número cada vez maior de pessoas nessa faixa etária que necessitam de uma comunicação mais efetiva justifica o desenvolvimento de estudos que se aprofundem no tema. Com o olhar voltado para a complexidade da comunicação dos idosos, o conhecimento sobre características da presbifonia em diferentes faixas etárias a partir dos 60 anos irá nortear o desenvolvimento de ações mais específicas para minimizar o declínio vocal e seus efeitos na qualidade de vida de idosos. O objetivo deste estudo foi verificar possíveis diferenças em aspectos vocais perceptivo-auditivos e acústicos em idosos com diagnóstico de presbifonia divididos em três faixas etárias.

\section{MÉTODO}

Trata-se de estudo transversal observacional retrospectivo, aprovado pelo Comissão de Ética para Análise de Projetos de Pesquisa do Hospital das Clínicas da Faculdade de Medicina da Universidade de São Paulo (CAAE 57164516.3.0000.0068). Foram selecionados, no banco de dados do ambulatório de voz da instituição, todos os indivíduos com idades a partir de 60 anos, cujo diagnóstico otorrinolaringológico, indicado no prontuário, fosse de presbifonia, e que tivessem realizado gravação da voz. Os fatores de exclusão foram a presença de comorbidades de natureza neurológica, auditiva, laríngea ou respiratória, além de histórico prévio de alterações vocais, de fala ou de linguagem. Todos os participantes assinaram Termo de Consentimento Livre e Esclarecido na ocasião da coleta dos dados.

Foram coletadas informações de 60 idosos, $36(60 \%)$ mulheres e 24 (40\%) homens, idades entre 60 e 90 anos (média de 72,3 anos). Para análise dos dados os participantes foram divididos em três grupos, de acordo com a faixa etária:

- G1: idades entre 60 e 70 anos; 27 participantes, média de 66,3 anos de idade [mínimo 65,19; máximo 67,44; desvio padrão 3,11]; 16 mulheres e 11 homens;

- G2: idades entre 71 e 80 anos; 25 participantes, média de 74,8 anos de idade [mínimo 73,84; máximo 75,96; desvio padrão 2,76]; 15 mulheres e 10 homens);

- G3: idades entre 81 e 90 anos; 8 participantes, média de 84,2 anos de idade [82,13, 86,63; desvio padrão 3,5]; 5 mulheres e 3 homens.

A gravação da voz dos pacientes ocorre na rotina dos atendimentos do ambulatório, em sala acusticamente tratada, sendo utilizado computador desktop (HP, EUA), software Audacity (equipe Audacity, EUA), interface Edirol UA-101 (Roland, Reino Unido) e microfone AKG modelo 520 (AKG, Alemanha). No roteiro de avaliação é utilizada a adaptação brasileira do Consensus Auditory-Perceptual Evaluation of Voice $(\mathrm{CAPE}-\mathrm{V})^{(11)}$. Como proposto no CAPE-V ${ }^{(12)}$, o grau de desvio é indicado em uma escala analógico-visual de 100 milímetros a 
partir da escuta das gravações de todas as tarefas vocais - emissão de vogais, leitura de frases e fala espontânea. A partir dessas orientações ${ }^{(12)}$ a análise do grau geral de disfonia, rugosidade, soprosidade, tensão, pitch e loudness foi realizada por uma fonoaudióloga especialista em voz, com mais de dez anos de experiência na utilização deste instrumento e na análise de vozes de idosos, e que apresentou alta confiabilidade intrajuiz (coeficiente de correlação intraclasse de 0,975).

A análise vocal acústica foi realizada com uso do software Voxmetria 2.6 (CTS Informática, Brasil). A emissão da vogal sustentada / $\varepsilon$ / durante cerca de cinco segundos, produzida em frequência e intensidade habituais, foi editada para utilização do terço medial, mais estável. Foram extraídas, no módulo de análise da qualidade vocal, as medidas de frequência fundamental (mínima, média, máxima e variabilidade), jitter, shimmer, glottal to noise excitation (proporção GNE), irregularidade e ruído. Foram consideradas as médias e, para interpretação dos dados, a classificação em normal/alterado segundo parâmetros de normalidade fornecidos pelo software: jitter PPQ entre 0,0 e 0,6\%, shimmer EPQ entre 0,0 e 6,5\%, proporção GNE entre 0,5 e 1,0, irregularidade entre 0,0 e 4,75 e ruído entre 0,0 e 2,5.

A mesma vogal foi utilizada para posicionar a produção vocal nos quadrantes do Diagrama de Desvio Fonatório (DDF), sendo o quadrante 1 considerado no software como a região de normalidade. Para a análise espectrográfica foi utilizada novamente a vogal / $\varepsilon /$ e considerada a presença/ausência dos seguintes aspectos: instabilidade, sub-harmônico, ruído em frequências agudas, ruído em frequências graves, quebra de frequência e quebra de sonoridade. A série de harmônicos foi classificada em adequada ou alterada.

Para extrair os valores relativos à intensidade vocal (mínima, média e máxima) foi utilizado o módulo de Análise de Voz do mesmo software no qual é requisitado o uso de algum trecho de fala. Foi selecionada aleatoriamente a frase "Érica tomou suco de pêra e amora" que contém todas as vogais do português brasileiro e é a primeira lida pelos indivíduos no momento da gravação de tarefas vocais. A análise descritiva considerou os dados relativos à idade e sexo e as medidas de frequência fundamental.

Para definição da análise estatística inferencial foi aplicado inicialmente o teste de normalidade de Kolmogorov-Smirnov. Para comparação entre os três grupos em relação às variáveis numéricas foi aplicado ou o teste não paramétrico de KruskallWallis ou o teste paramétrico ANOVA One-Way, usando comparações múltiplas de Tukey para analisar as diferenças significativas duas a duas, quando necessário. Para comparação quanto às variáveis categóricas foi aplicado o teste exato de Fisher. O nível de significância adotado foi de 5\%.

\section{RESULTADOS}

Ao se considerar inicialmente todos os idosos, independentemente dos grupos etários, destacaram-se as seguintes médias: grau geral de disfonia de 52,5 e de rugosidade 48,8, jitter $0,75 \%$, intensidade média 59,9 dB, série de harmônicos alterada $(100,0 \%)$, ruído em frequências graves $(96,7 \%)$, instabilidade $(82,8 \%)$, subharmônicos $(58,3 \%)$ e ruído em frequências agudas $(56,7 \%)$.

Na comparação entre os grupos, os idosos do G3 apresentaram maior soprosidade e desvio de pitch $(\mathrm{p}=0,025, \mathrm{p}=0,018)$ que o $\mathrm{G} 1$; valores mais elevados de shimmer $(\mathrm{p}=0,017)$ que o $\mathrm{G} 2$; e maior irregularidade que G1 e G2 ( $\mathrm{p}=0,042, \mathrm{p}=0,011)$ (Tabela 1). No G3 a maioria dos participantes apresentou DDF alterado e também quebra de frequência, o que não se observou em G1 e G2 (Tabela 2).

Tabela 1. Comparação entre os idosos dos três grupos etários em relação às variáveis numéricas decorrentes das análises perceptivo-auditiva e acústica

\begin{tabular}{|c|c|c|c|c|c|c|}
\hline \multirow{2}{*}{\multicolumn{2}{|c|}{ Aspectos analisados }} & \multicolumn{3}{|c|}{ Grupos } & \multirow{2}{*}{ Valor de $p$} & \multirow{2}{*}{$\begin{array}{l}\text { Comparações } \\
\text { Múltiplas 2x2 }\end{array}$} \\
\hline & & \multirow{2}{*}{$\begin{array}{c}\mathrm{G} 1(\mathrm{~N}=27) \\
46,7\end{array}$} & \multirow{2}{*}{$\frac{\mathrm{G} 2(\mathrm{~N}=25)}{50,1}$} & \multirow{2}{*}{$\frac{\mathrm{G} 3(\mathrm{~N}=8)}{60,6}$} & & \\
\hline Grau geral & Média & & & & & \\
\hline & Mediana & 44,0 & 53,0 & 58,5 & 0,179 & \\
\hline & DP & 19,2 & 17,4 & 18,5 & & \\
\hline \multirow[t]{2}{*}{ Rugosidade } & Média & 42,7 & 44,4 & 59,3 & & \\
\hline & Mediana & 40,0 & 42,0 & 58,5 & 0,110 & \\
\hline \multirow{2}{*}{ Soprosidade } & Mediana & 32,0 & 32,0 & 52,5 & $0,029^{*}$ & $(71-80) \times(81-90)(p)=0,054$ \\
\hline & $\mathrm{DP}$ & 21,4 & 20,9 & 21,0 & & \\
\hline \multirow[t]{3}{*}{ Tensão } & Média & 21,7 & 18,6 & 27,0 & & \\
\hline & Mediana & 18,0 & 8,0 & 35,5 & 0,625 & \\
\hline & DP & 23,3 & 22,2 & 23,4 & & \\
\hline \multirow[t]{3}{*}{ Loudness } & Média & 17,2 & 21,1 & 32,0 & & \\
\hline & Mediana & 0,0 & 22,0 & 37,0 & 0,280 & \\
\hline & DP & 27,1 & 23,2 & 29,9 & & \\
\hline
\end{tabular}

*estatisticamente significativo; Teste ANOVA One-way ou Kruskal-Wallis

Legenda: DP = desvio padrão; GNE = Glottal-to-Noise Excitation Ratio 
Tabela 1. Continuação...

\begin{tabular}{|c|c|c|c|c|c|c|}
\hline \multirow{2}{*}{\multicolumn{2}{|c|}{ Aspectos analisados }} & \multicolumn{3}{|c|}{ Grupos } & \multirow{2}{*}{ Valor de $p$} & \multirow{2}{*}{$\begin{array}{l}\text { Comparações } \\
\text { Múltiplas 2x2 } \\
\end{array}$} \\
\hline & & \multirow{2}{*}{$\begin{array}{c}\mathrm{G} 1(\mathrm{~N}=27) \\
0,72\end{array}$} & \multirow{2}{*}{$\begin{array}{c}\mathrm{G} 2(\mathrm{~N}=25) \\
0,53\end{array}$} & \multirow{2}{*}{$\frac{\mathrm{G} 3(\mathrm{~N}=8)}{1,01}$} & & \\
\hline Jitter & Média & & & & & \\
\hline & Mediana & 0,31 & 0,17 & 0,89 & 0,482 & \\
\hline & $\mathrm{DP}$ & 1,26 & 0,66 & 0,76 & & \\
\hline Shimmer & Média & 5,01 & 4,25 & 8,49 & & $(60-70) \times(81-90)(p)=0,058$ \\
\hline & Mediana & 3,95 & 3,47 & 7,62 & $0,010^{\star}$ & $(71-80) \times(81-90)(p)=0,017^{*}$ \\
\hline Irregularidade & Média & 3,76 & 3,52 & 4,85 & & $(60-70) \times(81-90)(p)=0,042^{*}$ \\
\hline & Mediana & 3,63 & 3,43 & 4,71 & $0,015^{\star}$ & $(71-80) \times(81-90)(p)=0,011^{*}$ \\
\hline & $\mathrm{DP}$ & 1,23 & 0,99 & 0,83 & & \\
\hline Medida GNE & Média & 0,71 & 0,72 & 0,58 & & \\
\hline & Mediana & 0,78 & 0,80 & 0,63 & 0,201 & \\
\hline & $\mathrm{DP}$ & 0,94 & 0,85 & 0,91 & & \\
\hline Intensidade média & Média & 60,5 & 59,4 & 59,8 & & \\
\hline & Mediana & 58,8 & 57,5 & 59,2 & 0,777 & \\
\hline & $\mathrm{DP}$ & 6,2 & 5,4 & 6,1 & & \\
\hline Intensidade & Média & 77,9 & 77,5 & 78,0 & & \\
\hline máxima & Mediana & 78,1 & 76,0 & 77,9 & 0,972 & \\
\hline & $\mathrm{DP}$ & 7,2 & 5,4 & 4,8 & & \\
\hline Intensidade & Média & 33,0 & 27,6 & 31,9 & & \\
\hline mínima & Mediana & 34,4 & 24,0 & 30,0 & 0,069 & \\
\hline
\end{tabular}

*estatisticamente significativo; Teste ANOVA One-way ou Kruskal-Wallis

Legenda: DP = desvio padrão; GNE = Glottal-to-Noise Excitation Ratio

Tabela 2. Comparação entre os idosos dos três grupos etários em relação às variáveis categóricas

\begin{tabular}{|c|c|c|c|c|c|c|c|c|c|c|}
\hline & & \multicolumn{6}{|c|}{ Grupos } & \multirow{2}{*}{\multicolumn{2}{|c|}{$\begin{array}{c}\text { Total } \\
(\mathrm{N}=60)\end{array}$}} & \multirow{3}{*}{ Valor de $\mathrm{p}$} \\
\hline \multicolumn{2}{|c|}{ Aspectos analisados } & \multicolumn{2}{|c|}{$\mathrm{G} 1(\mathrm{~N}=27)$} & \multicolumn{2}{|c|}{$\mathrm{G} 2(\mathrm{~N}=25)$} & \multicolumn{2}{|c|}{ G3 $(\mathrm{N}=8)$} & & & \\
\hline & & $\mathrm{N}$ & $\%$ & $\mathrm{~N}$ & $\%$ & $\mathrm{~N}$ & $\%$ & $\mathrm{~N}$ & $\%$ & \\
\hline Sexo & Feminino & 16 & 59,3 & 15 & 60,0 & 5 & 62,5 & 36 & 60,0 & $>0,999$ \\
\hline \multirow[t]{2}{*}{ DDF } & alterado & 11 & 40,7 & 7 & 28,0 & 5 & 62,5 & 23 & 38,3 & 0,235 \\
\hline & normal & 16 & 59,3 & 18 & 72,0 & 3 & 37,5 & 37 & 61,7 & \\
\hline \multirow[t]{2}{*}{ Sub harmônico } & ausente & 11 & 40,7 & 10 & 40,0 & 4 & 50,0 & 25 & 41,7 & 0,876 \\
\hline & presente & 16 & 59,3 & 15 & 60,0 & 4 & 50,0 & 35 & 58,3 & \\
\hline \multirow[t]{2}{*}{ ruído freq agudas } & ausente & 12 & 44,4 & 10 & 40,0 & 4 & 50,0 & 26 & 43,3 & 0,880 \\
\hline & presente & 15 & 55,6 & 15 & 60,0 & 4 & 50,0 & 34 & 56,7 & \\
\hline ruído freq graves & ausente & 1 & 3,7 & 1 & 4,0 & 0 & ,0 & 2 & 3,3 & $>0,999$ \\
\hline quebra de frequencia & presente & 10 & 37,0 & 10 & 40,0 & 5 & 62,5 & 25 & 41,7 & \\
\hline \multirow[t]{2}{*}{ quebra de sonoridade } & ausente & 23 & 85,2 & 21 & 84,0 & 7 & 87,5 & 51 & 85,0 & $>0,999$ \\
\hline & presente & 4 & 14,8 & 4 & 16,0 & 1 & 12,5 & 9 & 15,0 & \\
\hline
\end{tabular}

Teste exato de Fisher

Legenda: DDF = Diagrama de Desvio Fonatório; NC = não calculável 


\section{DISCUSSÃO}

Analisar diferenças na voz de idosos de idades variadas, de ambos os sexos, com diagnóstico de presbifonia e sem comorbidades que poderiam impactar na produção vocal foi a proposta deste estudo. Os idosos dos três grupos apresentaram alterações vocais perceptivo-auditivas e acústicas descritas na literatura, decorrentes das mudanças fisiológicas, e que se interrelacionam, como rugosidade, soprosidade, jitter elevado, série de harmônicos alterada, instabilidade, ruído em frequências graves e agudas, presença de sub-harmônicos e intensidade vocal reduzida ${ }^{(1,4,5,7,9,13)}$. Estudo com idosas coristas e não coristas encontrou alterações perceptivo-auditivas em menor grau ${ }^{(14)}$.

$\mathrm{Na}$ comparação entre os grupos foi possível verificar pior grau de soprosidade e pitch mais alterado na faixa etária acima de 80 anos em relação aos idosos de 60 a 70 anos, indicando maior impacto das mudanças fisiológicas ${ }^{(1,3-5,7)}$ nos mais velhos. Idosos com presbifonia, de diferentes idades, se diferenciaram de idosos sem presbifonia em todos os aspectos propostos no CAPE-V, exceto pitch $^{(6)}$. Considerando os parâmetros de normalidade do software utilizado, a média de jitter do G3 foi a mais alterada, com G1 e G2 ficando no limite da normalidade, mas vale destacar que o programa não explicita se este parâmetro se aplica a idosos ou apenas a adultos.

Algumas outras alterações comumente descritas em aspectos como jitter, shimmer e irregularidade ${ }^{(1,7)}$ foram observadas apenas no grupo de idosos com mais de 80 anos de idade, o que pode indicar que o impacto da redução de amplitude da onda mucosa, da irregularidade de vibração das pregas vocais e do espessamento e arqueamento das pregas vocais observados nos idosos ${ }^{(1,4,5,7,9)}$ é maior nesta faixa. Idosos com presbifonia podem apresentar shimmer alterado em relação a idosos sem presbifonia, independentemente da idade ${ }^{(6)}$. Este maior impacto das mudanças fisiológicas nos idosos com mais idade também pode justificar a proporção GNE no limite da normalidade e alteração no DDF e quebra de frequência na maioria dos idosos deste grupo. A impossibilidade de adução completa das pregas vocais durante a fonação pode interferir na estabilidade e controle de frequência ${ }^{(15)}$. De modo geral foi possível verificar deterioração de alguns aspectos apenas nos idosos com idades acima de 80 anos, assim como algumas alterações com maior gravidade.

Este estudo teve como limitação o comparativo entre sexos, o que pode ter impactado na comparação entre os grupos em relação ao aspecto pitch. Pelas diferenças anatomofisiológicas entre homens e mulheres, como a menor amplitude do movimento muco ondulatório das pregas vocais nos homens idosos em relação às mulheres ${ }^{(13)}$, pretende-se, com a continuidade deste estudo, analisar todos os aspectos separadamente por sexo. Além disso, devem ser investigados fatores relacionados ao uso profissional da voz e consumo de medicamentos que podem impactar negativamente na $\mathrm{voz}^{(1)}$.

Esta pesquisa se diferencia de outras pela participação apenas de idosos com diagnóstico de presbifonia e ausência de comorbidades que poderiam impactar na fonação. Essa escolha, se por um lado dificultou a composição da amostra, por outro evitou vieses pela presença de outras doenças que poderiam levar a maior degradação vocal. Estudo que comparou idosos com e sem presbifonia observou relação entre a presença do distúrbio e menor nível de atividade física, níveis de estresse mais elevados, hiperfunção vocal e menor qualidade de vida ${ }^{(6)}$.

A fonoterapia pode impactar positivamente na performance vocal e comunicação social do idoso, bem como melhorar sua qualidade de vida ${ }^{(1)}$. Por isso, é importante a identificação precoce desses indivíduos com maior risco de problemas vocais em função da idade ${ }^{(6)}$. O acompanhamento longitudinal de idosos com e sem presbifonia submetidos ou não à intervenção fonoaudiológica possibilitaria entender os benefícios para esses idosos, especialmente a partir dos 80 anos.

Numa sociedade em que a expectativa de vida aumenta, propiciar melhores condições comunicativas a esses idosos é de extrema importância. Assim, os achados se mostraram relevantes e poderão ser considerados no planejamento de ações específicas para esta população de acordo com a faixa etária com objetivo de retardar o declínio vocal.

\section{CONCLUSÃO}

Diversos aspectos perceptivo-auditivos e acústicos se apresentaram mais desviados nos participantes com mais idade, o que reforça a necessidade de serem consideradas essas especificidades tanto na avaliação dos efeitos do envelhecimento na voz quanto no desenvolvimento de ações para minimizar o declínio vocal.

\section{REFERÊNCIAS}

1. Mezzedimi C, Di Francesco M, Livi W, Spinosi MC, De Felice C. Objective evaluation of presbyphonia: spectroacoustic study on 142 patients with Praat. J Voice. 2017;31(2):257.e25-32. http://dx.doi.org/10.1016/j. jvoice.2016.05.022. PMid:27427181.

2. Wong HYK, Ma EPM. Self-Perceived voice problems in a nontreatment seeking older population in Hong Kong. J Voice. 2021;35(4):597-603. http://dx.doi.org/10.1016/j.jvoice.2019.12.012. PMid:31911022.

3. Gregory ND, Chandran S, Lurie D, Sataloff RT. Voice Disorders in the elderly. J Voice. 2012;26(2):254-8. http://dx.doi.org/10.1016/j.jvoice.2010.10.024. PMid:21530163.

4. Kendall K. Presbyphonia: a review. Curr Opin Otolaryngol Head Neck Surg. 2007;15(3):137-40. http://dx.doi.org/10.1097/MOO.0b013e328166794f PMid:17483679.

5. Kost KM, Sataloff RT. Voice disorders in the elderly. Clin Geriatr Med. 2018;34(2):191-203. http://dx.doi.org/10.1016/j.cger.2018.01.010. PMid:29661332.

6. Angadi V, McMullen C, Andreatta R, Dietrich M, Uhl T, Stemple J. Biobehavioral measures of Presbylaryngeus. J Voice. 2020;34(3):415-25. http://dx.doi.org/10.1016/j.jvoice.2018.11.005. PMid:30503609.

7. Martins RH, Gonçalvez TM, Pessin AB, Branco A. Aging voice: presbyphonia Aging Clin Exp Res. 2014;26(1):1-5. http://dx.doi.org/10.1007/s40520013-0143-5. PMid:24068559.

8. Menezes LN, Vicente LCC. Envelhecimento vocal em idosos instucionalizados. Rev CEFAC. 2007;9(1):90-8. http://dx.doi.org/10.1590/ S1516-18462007000100012.

9. Gois ACB, Pernambuco LA, Lima KC. Factors associated with voice disorders among the elderly: a systematic review. Braz J Otorhinolaryngol. 2018;84(4):506-13. http://dx.doi.org/10.1016/j.bjorl.2017.11.002. PMid:29331352.

10. Miranda GMD, Mendes ACG, Silva ALA. Population aging in Brazil: current and future social challenges and consequences. Rev Bras Geriatr Gerontol. 2016;19(3):507-19. http://dx.doi.org/10.1590/1809-98232016019.150140. 
11. Behlau M, Rocha B, Englert M, Madazio G. Validation of the Brazilian Portuguese CAPE-V Instrument-Br CAPE-V for Auditory-Perceptual Analysis. J Voice. 2020. In press. http://dx.doi.org/10.1016/j.jvoice.2020.07.007. PMid:32811691.

12. ASHA: American Speech-Language-Hearing Association. Consensus Auditory-Perceptual Evaluation of Voice (CAPE-V). ASHA Special Interest Group 3, Voice and Voice Disorders [Internet]. Washington: ASHA; 2009 [citado em 2020 Abr 24]. Disponível em: https://www.asha. org/uploadedFiles/ASHA/SIG/03/CAPE-V-Procedures-and-Form.pdf

13. Sulter AM, Schutte HK, Miller DG. Standardized laryngeal videostroboscopic rating: differences between untrained and trained male and female subjects, and effects of varying sound intensity, fundamental frequency and age. JVoice. 1996;10(2):17589. http://dx.doi.org/10.1016/S0892-1997(96)80045-2. PMid:8734393.
14. Aquino FS, Silva MA, Teles LC, Ferreira LP. Características da voz falada de idosas com prática de canto coral. CoDAS. 2016;28(4):446-53. http:// dx.doi.org/10.1590/2317-1782/20162015109. PMid:27652927.

15. Meirelles RC, Bak R, Cruz FC. Presbifonia. Revista do Hospital Universitário Pedro Ernesto. 2012;11:77-82.

\section{Contribuição dos autores}

ABPG foi responsável pela organização e análise dos dados e pela escrita do artigo; MSZ participou da análise dos dados e discussão dos resultados e foi responsável pela correção e finalização do artigo; $K N$ foi responsável pelo delineamento e coordenação do estudo, pela análise e discussão dos dados e revisão do manuscrito. 